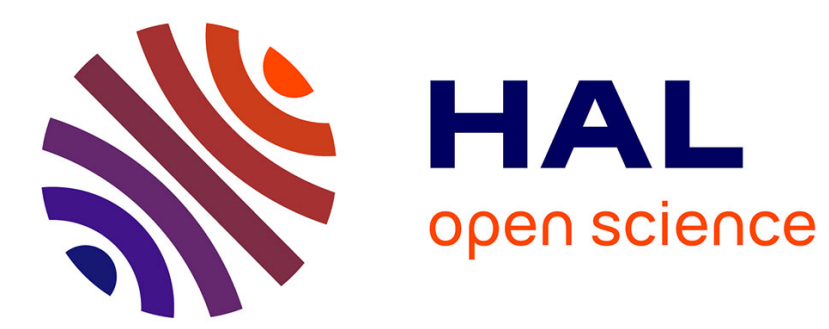

\title{
PROJECTION BASED APPROACH FOR REFLECTION SYMMETRY DETECTION
}

Thanh Phuong Nguyen

\section{To cite this version:}

Thanh Phuong Nguyen. PROJECTION BASED APPROACH FOR REFLECTION SYMMETRY DETECTION. ICIP, Sep 2019, Taipei, Taiwan. hal-02133544

\section{HAL Id: hal-02133544 \\ https://hal.science/hal-02133544}

Submitted on 18 May 2019

HAL is a multi-disciplinary open access archive for the deposit and dissemination of scientific research documents, whether they are published or not. The documents may come from teaching and research institutions in France or abroad, or from public or private research centers.
L'archive ouverte pluridisciplinaire HAL, est destinée au dépôt et à la diffusion de documents scientifiques de niveau recherche, publiés ou non, émanant des établissements d'enseignement et de recherche français ou étrangers, des laboratoires publics ou privés. 


\title{
PROJECTION BASED APPROACH FOR REFLECTION SYMMETRY DETECTION
}

\author{
Thanh Phuong Nguyen \\ Université de Toulon, CNRS, LIS, UMR 7020, 83957 La Garde, France \\ Aix-Marseille Université, CNRS, ENSAM, LIS, UMR 7020, 13397 Marseille, France
}

\begin{abstract}
A novel method for reflection symmetry detection is addressed using a projection-based approach that allows to deal effectively with additional noise, non-linear deformations, and composed shapes that are not evident for classic contourbased approaches. A new symmetry measure is also proposed to measure how good the detected symmetry is. Experiments validate the interest of our proposed method.
\end{abstract}

Index Terms - Reflection symmetry, Radon, $\mathcal{R}$-transform

\section{INTRODUCTION}

Symmetry is very popular in both artificial and natural scenes because most mand-made and biological objects have symmetric properties. In addition, symmetric structures are important visual features for human attention, therefore symmetry detection plays an important role in computer vision.

There are two main problems in symmetry detection. The first one aims at detecting and measuring the rotational symmetries in a shape. Lin et al. [1] proposed fold-invariant shape-specific points for detecting the orientations of rotationally symmetric shapes. Cornelius and Loy [2] detected planar rotational symmetry under affine projection. Prasad and Davis [3] localized multiple rotational symmetries in natural images using gradient magnitude field. Loy and Eklunhd [4] grouped symmetric pairs of feature points and characterizing the symmetries presented in an image. Flusser and Suk [5] introduced a new set of invariant moments for recognition of objects having $\mathrm{n}$-fold rotation symmetry. Yip introduced different methods using Hough transform [6] or Fourier descriptor [7] for the detection of rotational symmetry.

The second one groups the methods for reflection symmetry detection. Ogawa [8] used Hough transform to detect axis of symmetry in shapes of line drawing. Yip [9] then developed this approach to deal with both reflection symmetry and skew-symmetry. Lei and Wong [10] also used Hough transform for detecting and recovering the pose of a reflection and rotational symmetry from a single weak perspective image. Cornelius and Loy [11] detected bilateral symmetry in images under perspective projection by matching pairs of symmetric features. Nagar and Raman [12] proposed an energy minimization approach to detect multiple reflection symme- tries. They also introduced an another work [13] for detecting approximated reflection symmetry in a set of points using optimization on manifold. Kazhdan [14] used Fourier methods to detect and compute reflective symmetries. In [15], Derrode and Ghorbel applied Fourrier-Melin transform for rotation and reflection symmetry estimation. Kiryati and Gofman [16] converted reflection symmetry detection into a global optimization problem. Cornelius et al. [17] used local affine frames (LAFs) constructed on maximally stable extremal regions to improve the detection of symmetric objects under perspective distortion.

We address in this paper a new method for reflection symmetry detection using $\mathcal{R}$-transform. Based on the projection based approach, this can deal naturally with composed shapes, additional noise, and non-linear deformations.

\section{BASIC MATERIALS}

Let us recall some basic materials of Radon transform and $\mathcal{R}$-transform [18]. Those will be used in the next section to propose a new method for reflection symmetry detection.

\subsection{Radon transform}

Let $f \in \mathbb{R}^{2}$ be a 2D function and $L(\theta, \rho)=\left\{\mathbf{x} \in \mathbb{R}^{2} \mid\right.$ $\mathbf{x} \cdot \mathbf{n}(\theta)=\rho\}$ be a straight line in $\mathbb{R}^{2}$, where $\theta$ is the angle $L$ makes with the $y$ axis, $\mathbf{n}(\theta)=(\cos \theta, \sin \theta)$, and $\rho$ is the radial distance from the origin to $L$. The Radon transform [19] of $f$, denoted as $\mathcal{R}_{f}$, is a functional defined on the space of lines $L(\theta, \rho)$ by calculating the line integral along each line as follows.

$$
\mathcal{R}_{f}(\theta, \rho)=\int f(\mathbf{x}) \delta(\rho-\mathbf{x} \cdot \mathbf{n}(\theta)) \mathrm{d} \mathbf{x}
$$

In shape analysis, the function $f$ is constrained to take value 1 if $\mathbf{x} \in \mathcal{D}$ and 0 otherwise, where $\mathcal{D}$ is the domain of the binary shape represented by $f$ (see Figure 1).

$$
f(\mathbf{x})=\left\{\begin{array}{l}
1 \text { if }(\mathbf{x}) \in \mathcal{D} \\
0 \text { otherwise }
\end{array}\right.
$$

Radon transform is robust to additive noise and has some interesting geometric properties [19] which are the base to 


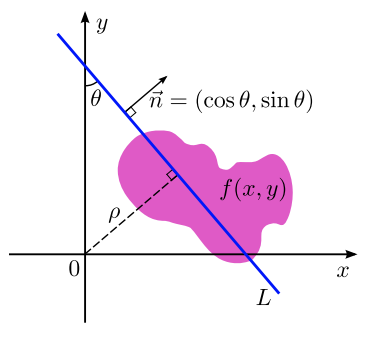

Fig. 1. Radon transform of a function $f(x, y)$

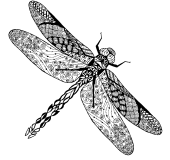

(a) Input

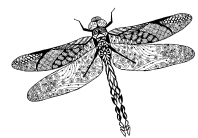

(b) Rotated

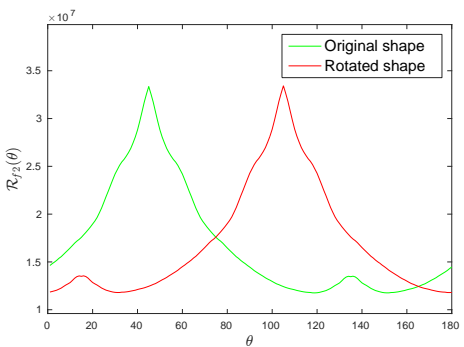

(c) $\mathcal{R}$-transforms
Fig. 2. Illustration of $\mathcal{R}$-transforms [18].

developpe an effective shape signature, namely $\mathcal{R}$-transform [18], presented in Section 2.2.

We also introduce the following notion that will be used latter. For each projection direction $\theta$, the radial distances $\rho_{1}^{\theta}$ and $\rho_{2}^{\theta}$ are respectively defined as $\rho_{1}^{\theta}=\inf \left\{\rho \mid \mathcal{R}_{\mathcal{D}}(\theta, \rho)>\right.$ $0\}$ and $\rho_{2}^{\theta}=\sup \left\{\rho \mid \mathcal{R}_{\mathcal{D}}(\theta, \rho)>0\right\}$. The "profile" (or Radon projection) of $\mathcal{D}$ in the direction $\theta$, denoted as $C_{\mathcal{D}}^{\theta}$, is defined as $\mathcal{R}_{\mathcal{D}}\left(\theta, \rho_{1}^{\theta}: \rho_{2}^{\theta}\right)$. More precisely, $C_{\mathcal{D}}^{\theta}\left(\rho-\rho_{1}^{\theta}\right)=$ $\mathcal{R}_{\mathcal{D}}(\theta, \rho), \forall \rho \in\left[\rho_{1}^{\theta}, \rho_{2}^{\theta}\right]$.

\section{2. $\mathcal{R}$-transform}

Tabbone et al. [18] introduced a transform, called $\mathcal{R}$ transform, for an effective shape representation as follows.

$$
R_{f 2}(\theta)=\int_{-\infty}^{+\infty} \mathcal{R}_{f}^{2}(\theta, \rho) d \rho
$$

They have shown the following properties of this transform.

- Periodicity: $R_{f 2}$ is periodical with period of $\pi$.

- Rotation: A rotation of the image by an angle $\theta_{0}$ leads to a circular shift of $R_{f 2}$ of $\theta_{0}$.

- Translation: $R_{f 2}$ is invariant against translation

- Scaling: A scaling of $f$ implies only a scaling in the amplitude of $R_{f 2}$

Please refer to [18] for more illustrations about the robustness of $\mathcal{R}$-transform against similarity transforms (translation, rotation, scaling), and non-linear deformations. These properties make $\mathcal{R}$-transform useful for shape analysis. It is also a base for different shape descriptors [20, 21, 22].

\section{REFLECTION SYMMETRY DETECTION}

\section{1. $\mathcal{R}$-transform and reflection symmetry}

Let us consider an arbitrary shape $\mathcal{D}$. It has reflection symmetry if there is at least one line which splits the shape in half so that one side is the mirror image of the other. For simplicity, $\mathcal{D}$ is called reflectionally symmetric in direction $\theta$ if it is reflectionally symmetric and contains an axis of symmetry in that direction.

As we have pointed out in Section 2.2, $\mathcal{R}$-transform has been proven to be robust against additive noise, nonlinear deformations [18]. It is invariant against similarity transforms: translation, and rotation. In addition, it is also invariant against scaling if the transform is normalized by a scaling factor. Those beneficial properties suggest that $\mathcal{R}$-transform can be served as an useful tool for shape analysis.

We propose in this section an another interesting property of $\mathcal{R}$-transform for reflection symmetry detection. The main idea is to convert the problem of detecting and measuring reflection symmetry of an arbitrary shape $\mathcal{D}$ into measuring the reflection symmetry in its $\mathcal{R}$-transform. Due to [18], $R_{f 2}(\theta)$ is periodical of period $\pi$ with respect to $\theta$, it is sufficient to consider $\mathcal{R}$-transform only on the set of projections $\Theta=[0, \pi)$ or $\Theta=0^{0}, 1^{0}, \ldots, 179^{0}$ for relection symmetry detection.

Figure 2 shows the $\mathcal{R}$-transforms of two reflectionally symmetric shapes. Similarly, Figures 3 .b presents $\mathcal{R}$ transform of a synthetic shape which is an isosceles triangle in Figure 3.a. It should be noted that the studied shape contains evidently one axis of reflection symmetry in direction $O^{0}$ while its $\mathcal{R}$-transform has two reflection symmetries at directions 0 and $\frac{\pi}{2}$. In addition, Figure 3.d shows the Radon projection of $\mathcal{D}$ in two above directions. We could make some important following remarks from those Figures.

- $\mathcal{R}$-transform contains rich information about rotational symmetric properties of shapes. If $\mathcal{D}$ contains reflection symmetry, its $\mathcal{R}$-transform is also reflectionally symmetric.

- Each detected axis of reflection symmetry of $\mathcal{D}$ in direction $\theta_{0}$ leads to 2 reflection symmetries which separate by an interval of $\frac{\pi}{2}$. Those correspond generally to two orthogonal directions: $\theta_{0}$ and $\theta_{0}+\frac{\pi}{2}$. This comes from the fact that $\mathcal{R}$-transform treats equally all projection values in each direction.

- Between two above detected directions, the profile of Radon projection $\left(\mathcal{R}_{f}(\theta, \rho)\right)$ is also reflectionally symmetric in the direction $\theta_{0}$. For the direction $\theta_{0}+\frac{\pi}{2}$, it is reflectionally symmetric if and only if $\mathcal{D}$ contains also reflection symmetry in direction $\theta_{0}+\frac{\pi}{2}$. Indeed, contrariwise to $\mathcal{R}$-transform, Radon projection $\left(\mathcal{R}_{f}(\theta, \rho)\right)$ consider the distribution of projection values for each direction. $\mathcal{R}_{f}(\theta, \rho)$ presents the distribution of two 
symmetric parts of $\mathcal{D}$ in direction $\theta_{0}$ of symmetric axis, therefore is is also reflectionally symmetric. It is not the same in direction $\theta_{0}+\frac{\pi}{2}$ because $\mathcal{D}$ is not reflectionally symmetric in this direction. Hence, it brings out the direction containing reflection symmetry. This allows to eliminate false reflection symmetry detection of a shape by considering its $\mathcal{R}$-transform.

\subsection{Proposed method for symmetry detection}

The analysis in Section 3.1 suggest an effective algorithm for reflection symmetry detection of a shape by considering its $\mathcal{R}$-transform. The main idea is to measure the similarity between its forward and backward circular shifts of each angle $\theta_{0}$. If this similarity is perfect, angle $\theta_{0}$ is candidate for a reflectionally symmetric direction. This can be confirmed by verifying if the Radon projection in this direction $\left(C_{\mathcal{D}}^{\theta_{0}}\right)$ is symmetric. This property can be checked simply by calculating the similarity between it and its inversion $\left(I\left(C_{\mathcal{D}}^{\theta_{0}}\right)\right)$. We propose to use Pearson's Linear Correlation Coefficient [23] to measure those similarities. For the simplicity, let us consider two vectors $X$, and $Y$ having a same dimensionality. The similarity between them is defined by function $\Omega$ as follows.

$$
\Omega(X, Y)=\frac{\sum_{j=1}^{n}(X-\bar{X})(Y-\bar{Y})}{\left\{\sum_{i=1}^{n}(X-\bar{X})^{2} \sum_{i=1}^{n}(Y-\bar{Y})^{2}\right\}^{1 / 2}}
$$

,where $n$ is the number of element in vector $X$, and $Y ; \bar{X}$, and $\bar{Y}$ are respectively mean values of $X$, and $Y$. Values of the correlation coefficient can range from -1 to +1 . A value of -1 indicates perfect negative correlation, while a value of +1 indicates perfect positive correlation. A value of 0 indicates no correlation between the columns.

Algorithm 1 addresses the proposed method for reflection symmetry detection by using some notations presented in Definition 1. Threshold $\tau$ is empirically set to 0.75 to simply remove non-signiticative candidates.

Definition 1 Given $x=\left\{x_{1}, x_{2}, \ldots, x_{n}\right\}$, a vector of $n$ elements. $F_{\zeta}^{i}(x)\left(\right.$ resp. $\left.B_{\zeta}^{i}(x)\right)$, which is called forward (resp. backward) circular shift of $x$ of step $i$, is defined as follows: $F_{\zeta}^{i}(x)=\left\{x_{i}, x_{i+1}, \ldots, x_{n}, x_{1}, \ldots, x_{i-1}\right\}$ (resp. $B_{\zeta}^{i}(x)=$ $\left.\left\{x_{i}, x_{i-1}, \ldots, x_{1}, x_{n}, \ldots, x_{i+1}\right\}\right)$. Specially, the inversion of $x$, called $I(x)$, is defined by $I(x)=B_{\zeta}^{n}(x)$

Figure 3 illustrates how Algorithm 1 works. First, $\mathcal{R}$ transform (Figure 3.b) of the studied shape (Figure 3.a) is constructed. Then, the merit profile, presented in Figure 3.c, is calculated by measuring the similarity between the forward and backward circular shifts at each direction $\theta$ of $\mathcal{R}$ transform. This gives two peaks being closed to 1 at directions $0^{0}$ and $\frac{\pi}{2}$ as candidates of symmetric direction. A simple check using correlation is then applied on Radon projection of each candidate direction (Figure 3.d) allows to eliminate direction $\frac{\pi}{2}$ and to confirm that direction 0 contains axis
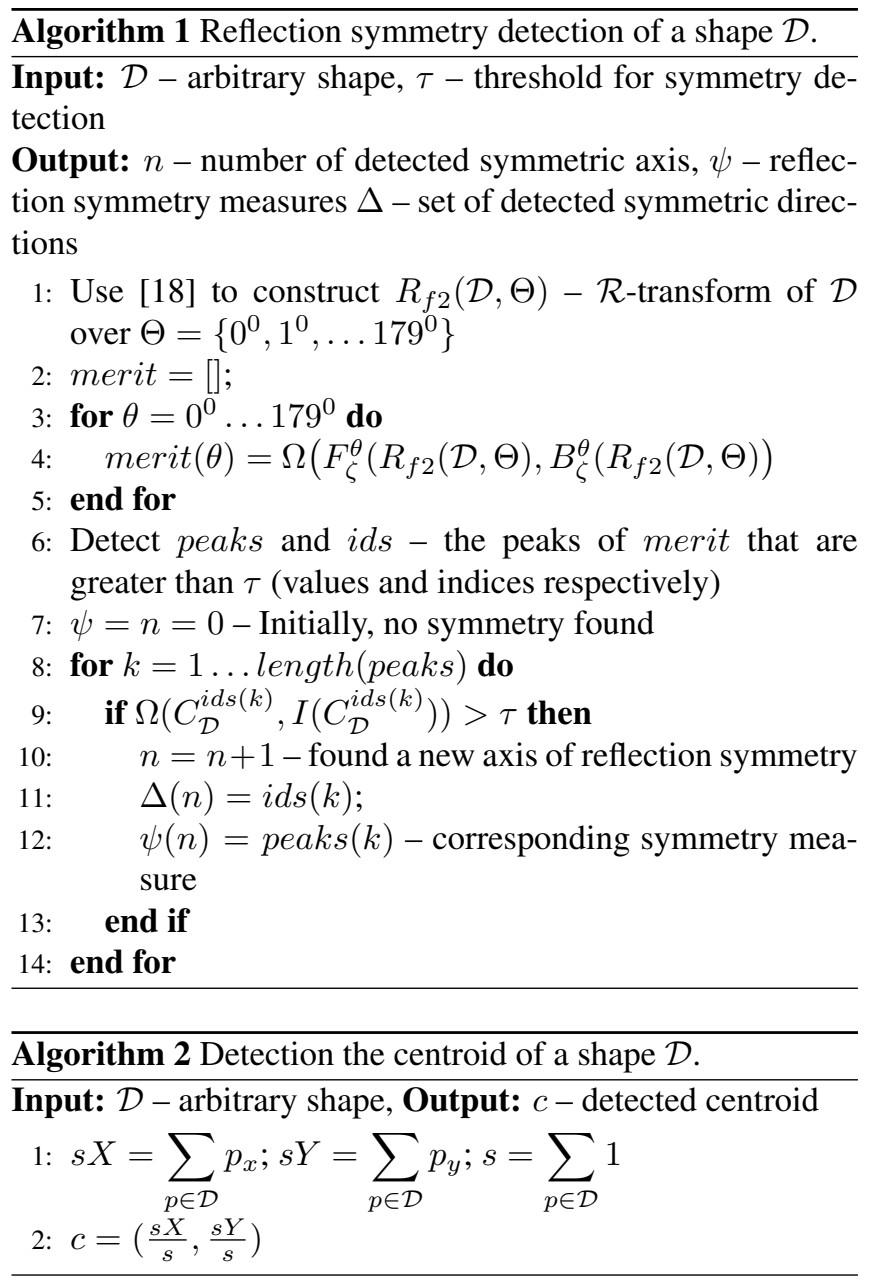

of reflection symmetry. In addition, the corresponding peak of merit profile defines how good the detection symmetry is. Therefore, is is proposed as measure of reflection symmetry at this direction. The more it closes to 1 , the more the symmetry is perfect. The corresponding symmetric axis is simply defined as the line of this direction and passes through the centroid of shape $\mathcal{D}$. Algorithm 2 recalls a well-known momentbased method to detect centroid of an arbitrary shape.

\section{EXPERIMENTS}

We first evaluate the proposed methods on synthetic shapes to verify its behavior. To do that, we create different regular polygons of which the size, orientation are varied. Figure 4 shows the obtained results with different synthetic shapes (i.e. arbitrary, and regular polygons). It could be noted that for 3-polygon and 5-polygon, the number of detected candidates on merit profile is twice the number of detected axis. A half of them have been removed after a simple verification to eliminate false symmetric axis. In addition, the first row of Figure 4 shows an experiment on a non-symmetric shape, its merit profile give highest value at only 0.59 , so no reflection 


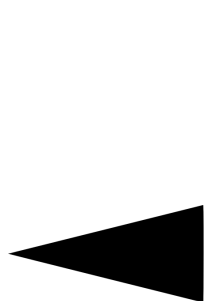

(a) Input

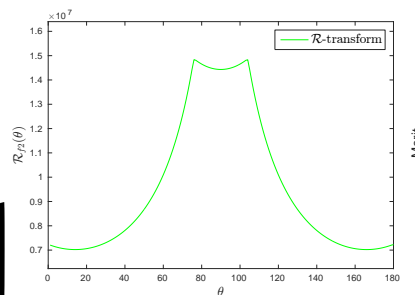

(b) $\mathcal{R}$-transform

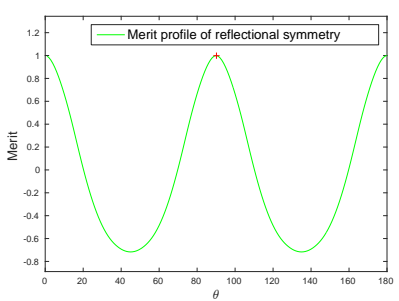

(c) Detect peaks of merit profile

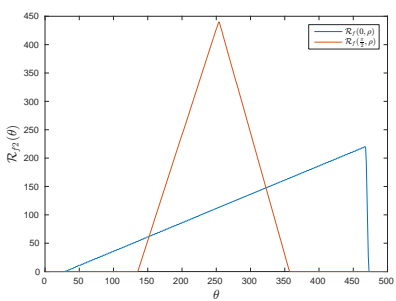

(d) Check Radon projections

Fig. 3. Different steps to detect reflection symmetry.

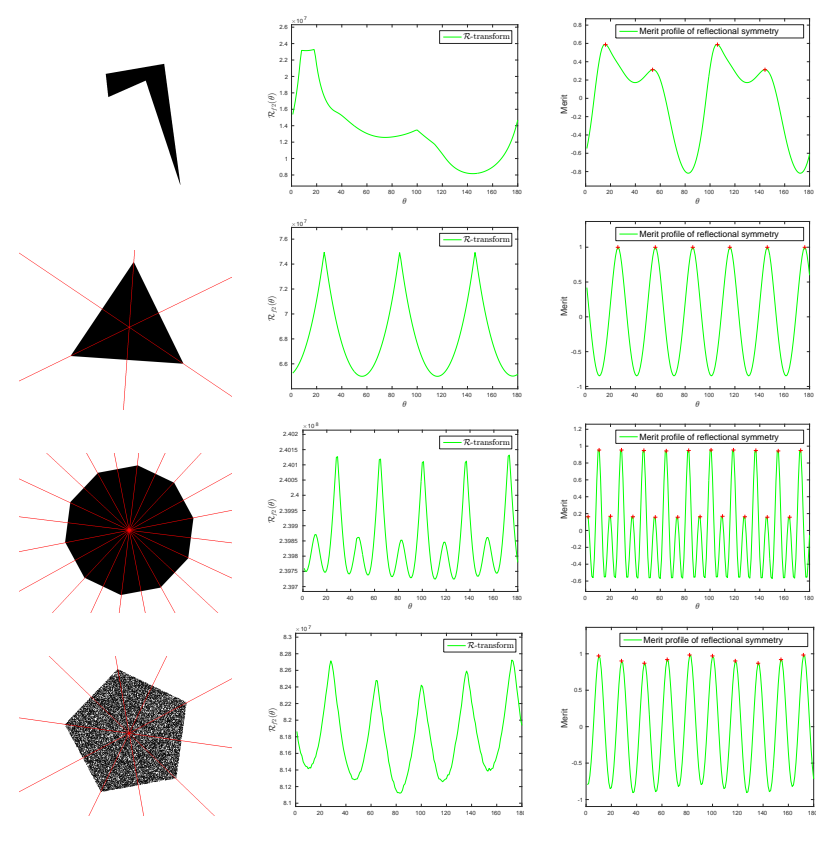

Fig. 4. Experiments on synthetic shapes.

symmetry is detection. The last row of Figure 4 presents an experiment on a heavy noisy regular polygon $(\mathrm{SNR}=1)$. Regarding its $\mathcal{R}$-transform, we can conclude that thanks to the the robustness of $\mathcal{R}$-transform against additional noise, and non-linear transform, the detection of symmetry detection is always robust in such conditions.

Figure 5 presents the results of our methods on real-world images. For each shape, a couple sets $(\Delta, \psi)$ determining the detected directions and estimated symmetry measures is shown. It could be said that the proposed method can deal with well with natural images thanks to the projection-based approach.

\section{CONCLUSIONS}

We have introduced a novel method for reflection symmetry detection based on $\mathcal{R}$-transform. It detect the symmetry axes and measure how good the symmetry is for each detected axis. Thanks to the beneficial properties of projection-based
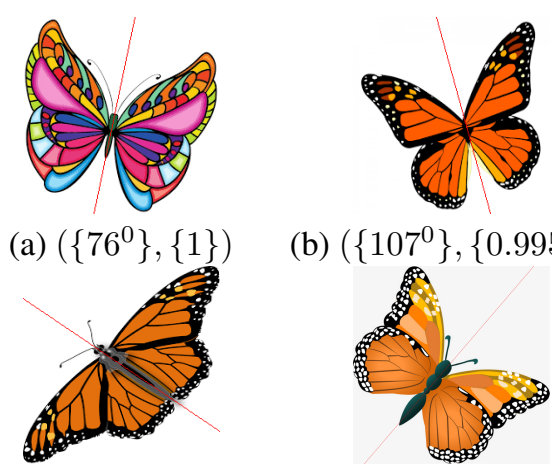

(b) $\left(\left\{107^{0}\right\},\{0.9957\}\right)$

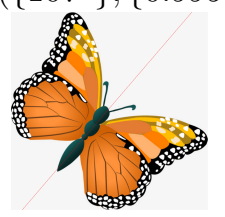

(c) $\left(\left\{141^{0}\right\},\{0.9994\}\right)$

(d) $\left(\left\{48^{0}\right\},\{0.9996\}\right)$

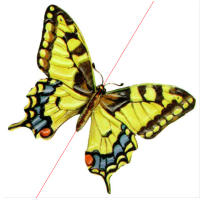

(e) $\left(\left\{58^{0}\right\},\{0.9916\}\right)$

Fig. 5. Experiments on realworld images: each shape is defined by the symmetry direction together with symmetry measure.

approach, the method can deal with addition noise, and nonlinear deformation. Moreover, it can process compounded shapes that are difficult for typical contour-based methods.

\section{ACKNOWLEDGMENT}

We would like to thank Dr. Thai V. Hoang for the fruitful discussions.

\section{REFERENCES}

[1] Ja-Chen Lin, Sheng-Lin Chou, and Wen-Hsiang Tsai, "Detection of rotationally symmetric shape orientations by fold-invariant shape-specific points," Pattern Recognition, vol. 25, no. 5, pp. 473-482, 1992.

[2] Hugo Cornelius and Gareth Loy, "Detecting rotational symmetry under affine projection," in $I C P R, 2006, \mathrm{pp}$. 292-295. 
[3] V. Shiv Naga Prasad and Larry S. Davis, "Detecting rotational symmetries," in ICCV, 2005, pp. 954-961.

[4] Gareth Loy and Jan-Olof Eklundh, "Detecting symmetry and symmetric constellations of features," in ECCV, Ales Leonardis, Horst Bischof, and Axel Pinz, Eds., 2006, vol. 3952 of LNCS, pp. 508-521.

[5] Jan Flusser and Tomás Suk, "Rotation moment invariants for recognition of symmetric objects," IEEE Transactions on Image Processing, vol. 15, no. 12, pp. 37843790, 2006.

[6] Raymond K. K. Yip, "A Hough transform technique for the detection of parallel projected rotational symmetry," Pattern Recognition Letters, vol. 20, no. 10, pp. 9911004, 1999.

[7] Raymond K. K. Yip, "Genetic Fourier descriptor for the detection of rotational symmetry," Image Vision Comput., vol. 25, no. 2, pp. 148-154, 2007.

[8] Hideo Ogawa, "Symmetry analysis of line drawings using the Hough transform," Pattern Recognition Letters, vol. 12, no. 1, pp. 9-12, 1991.

[9] Raymond K. K. Yip, "A Hough transform technique for the detection of reflectional symmetry and skewsymmetry," Pattern Recognition Letters, vol. 21, no. 2, pp. 117-130, 2000.

[10] Yiwu Lei and Kok Cheong Wong, "Detection and localisation of reflectional and rotational symmetry under weak perspective projection," Pattern Recognition, vol. 32, no. 2, pp. 167-180, 1999.

[11] Hugo Cornelius and Gareth Loy, "Detecting bilateral symmetry in perspective," in CVPR, 2006, p. 191.

[12] Rajendra Nagar and Shanmuganathan Raman, "Reflection symmetry axes detection using multiple model fitting," IEEE Signal Process. Lett., vol. 24, no. 10, pp. 1438-1442, 2017.

[13] Rajendra Nagar and Shanmuganathan Raman, "Detecting approximate reflection symmetry in a point set using optimization on manifold," IEEE Transactions on Signal Processing, 2019.

[14] Michael M. Kazhdan, Bernard Chazelle, David P. Dobkin, Adam Finkelstein, and Thomas A. Funkhouser, "A reflective symmetry descriptor," in ECCV, 2002, pp. 642-656.

[15] Stéphane Derrode and Faouzi Ghorbel, "Shape analysis and symmetry detection in gray-level objects using the analytical Fourier-Mellin representation," Signal Processing, vol. 84, no. 1, pp. 25-39, 2004.
[16] Nahum Kiryati and Yossi Gofman, "Detecting symmetry in grey level images: The global optimization approach," International Journal of Computer Vision, vol. 29, no. 1, pp. 29-45, 1998.

[17] Hugo Cornelius, Michal Perdoch, Jiri Matas, and Gareth Loy, "Efficient symmetry detection using local affine frames," in SCIA, Bjarne K. Ersbøll and Kim Steenstrup Pedersen, Eds., 2007, vol. 4522 of LNCS, pp. 152-161.

[18] Salvatore Tabbone, Laurent Wendling, and J.-P. Salmon, "A new shape descriptor defined on the Radon transform," Computer Vision and Image Understanding, vol. 102, no. 1, pp. 42-51, 2006.

[19] Stanley R. Deans, The Radon Transform and Some of Its Applications, Krieger Publishing Company, 1993.

[20] Thai V. Hoang and Salvatore Tabbone, "The generalization of the R-transform for invariant pattern representation," Pattern Recognition, vol. 45, no. 6, pp. 21452163, 2012.

[21] Thanh Phuong Nguyen and Thai V. Hoang, "Projectionbased polygonality measurement," IEEE Trans. Image Processing, vol. 24, no. 1, pp. 305-315, 2015.

[22] Thanh Phuong Nguyen and Xuan Son Nguyen, "Shape measurement using LIP-signature," Computer Vision and Image Understanding, vol. 171, pp. 83-94, 2018.

[23] Stephen M. Stigler, "Francis Galton's Account of the Invention of Correlation," Statistical Science, vol. 4, no. 2, pp. 73-79, 1989. 